\title{
Rene Policistico e trapianto di rene: accesso alla lista d'attesa e post trapianto: risultati della ricerca
}

Giornale di Tecniche Nefrologiche e Dialitiche 2019, Vol. 3I(3) 176-182

(C) The Author(s) 2019

Article reuse guidelines:

sagepub.com/journals-permissions DOI: 10.1 I 177/0394936219876868 journals.sagepub.com/home/gtn

(S) SAGE

\section{Rossana Caldara}

\begin{abstract}
Polycystic kidney disease and kidney transplantation: access to waiting list and post-transplant

Autosomal-dominant polycystic kidneydisease (ADPKD) is the leading genetic cause of end-stage renal disease (ESRD) worldwide. The number of ADPKD patients who are listed for transplantation or receive a kidney transplant is continuously increasing over time.

AIRP conducted a survey to investigate the ADPKD patient journey, meaning the personal experience and expectations of people regarding kidney transplantation as therapeutic option of end-stage renal failure.

The survey was conducted on $38 \mathrm{I}$ people with ADPKD, using computer-assisted web interviewing (CAWI).

The results confirm that there are problems that need to be addressed before listing an ADPKD patient for a kidney transplantation, namely the patient's comorbidities, the complexity of pre-transplant assessments and the shortage of organs. Pre-emptive transplantation from cadaver donor is a rare event in our country but it is a valid option, especially in case of living donation.

Immunosuppression is well tolerated in a high percentage of subjects, but a follow-up is necessary to monitor negative side effects.

Despite these problems, the outcome of kidney transplantation is optimal in these patients. Also, the relationship between patients and Nephrologists and/or Transplant Centers is important to ensure a positive outcome.
\end{abstract}

\section{Keywords}

ADPKD, Patient journey, Kidney transplantation, ESRD, Transplant Centers

La malattia da rene policistico autosomico dominante (autosomal-dominant polycystic kidney disease, ADPKD) è la prima patologia, geneticamente determinata, che porta all'insufficienza renale cronica, ed il numero dei pazienti che vengono inseriti in lista d'attesa di trapianto di rene è aumentata nel tempo.

Per quanto riguarda le casistiche italiane, dal gennaio 2002 al dicembre 2010, 1,588 pazienti affetti da ADPKD sono stati sottoposti ad un primo trapianto di rene, rappresentando il $13 \%$ di tutti i trapiantati di quel periodo. ${ }^{1}$

Un aspetto importante che è emerso dall'analisi di questa popolazione di pazienti è che i reni trapiantati nei pazienti con ADPKD presentano una durata nel tempo, sopravvivenza dell'organo, migliore rispetto a quella osservata in pazienti con altre patologie. ${ }^{1}$

Questa osservazione è stata confermata anche dai dati del registro europeo (ERA-EDTA) che dimostrano come, dopo 15 anni dal trapianto, il $76.6 \%$ dei pazienti ADPKD ha ancora un rene funzionante, mentre tale percentuale è più bassa per soggetti affetti da altre glomerulonefriti primarie. ${ }^{2}$

Il trapianto di rene rappresenta quindi un'opzione terapeutica valida da considerare quando oramai la malattia ha portato all'insufficienza renale ed alla necessità di trattamento sostitutivo (emodialisi o dialisi peritoneale).

Quando affronta il trapianto, il paziente passa attraverso diverse fasi, come quella dell'inserimento in lista, la fase del trapianto e la successiva fase di controllo nel tempo, a breve e lungo termine.

Durante queste fasi, il paziente ed i suoi familiari fanno riferimento ai propri nefrologi ed ai medici dei centri di trapianto. Esiste tuttavia una disomogeneità, sul territorio italiano, relativa alle modalità di come ciò avviene.

Per fotografare la realtà del nostro Paese, AIRP ha condotto un'indagine, presso i propri iscritti, per verificare alcuni aspetti relativi all'accesso alle liste di trapianto di rene e al monitoraggio clinico post-intervento a breve (primo anno post-trapianto) e lungo termine (dal secondo anno in poi).

Medicina Interna e dei Trapianti, Ospedale San Raffaele, Milano, Italy

\section{Corrispondenza:}

Dott.ssa Rossana Caldara, Medicina Interna e dei Trapianti, Ospedale

San Raffaele, via Olgettina 60, Milano 20162, Italy.

E-mail: caldara.rossana@hsr.it 


\section{Metodologia}

L'indagine è stata realizzata da AIRP onlus tramite metodologia CAWI (computer-assisted web interviewing) su 384 pazienti con ADPKD residenti in Italia. Il sondaggio è stato effettuato online dal 25/03/2019 al 30/04/2019, ed i canali di somministrazione sono stati la Newsletter e la pagina Facebook di AIRP.

\section{Principali risultati}

Alle domande del questionario hanno risposto 381 pazienti, di cui il $54.9 \%$ donne e $44.4 \%$ uomini. Tutte le fasce d'età sono state rappresentate, anche se la più presente è stata quella compresa tra i 46 ed i 55 anni. Il fatto che la fascia di età più rappresentata sia quella dell'età già più avanzata è verosimilmente legata al fatto che l'insufficienza renale, se pur nota sin dall'età giovanile, si sviluppa tardivamente nel corso della vita. Infatti, è già noto da tempo che nella maggior parte dei pazienti la funzione renale rimane preservata per un lungo periodo per poi ridursi abbastanza rapidamente dopo i 40 anni, giungendo all'insufficienza renale in circa 10 anni. $^{3}$

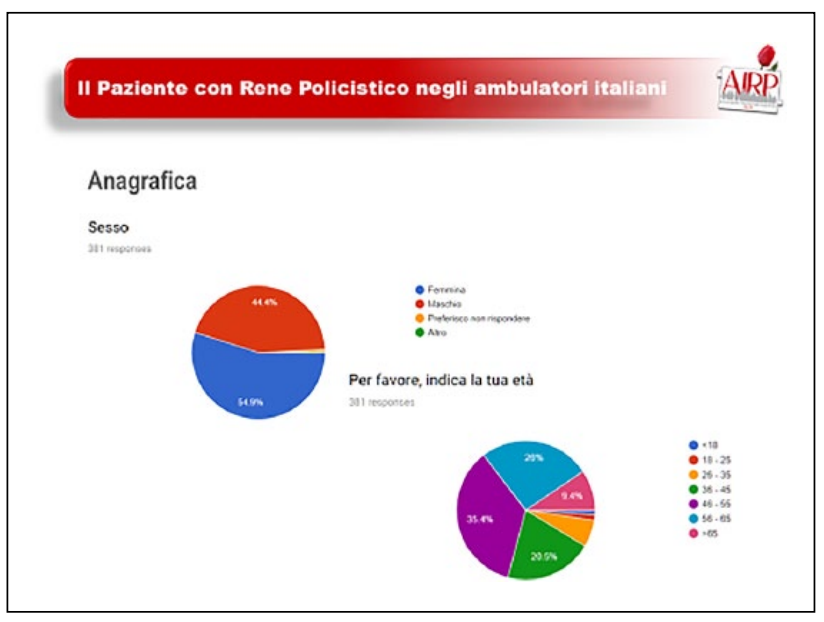

Le risposte sono giunte da tutte le Regioni del nostro Paese ed anche da stranieri seguiti dalle nostre Nefrologie e dai nostri Centri di trapianto.

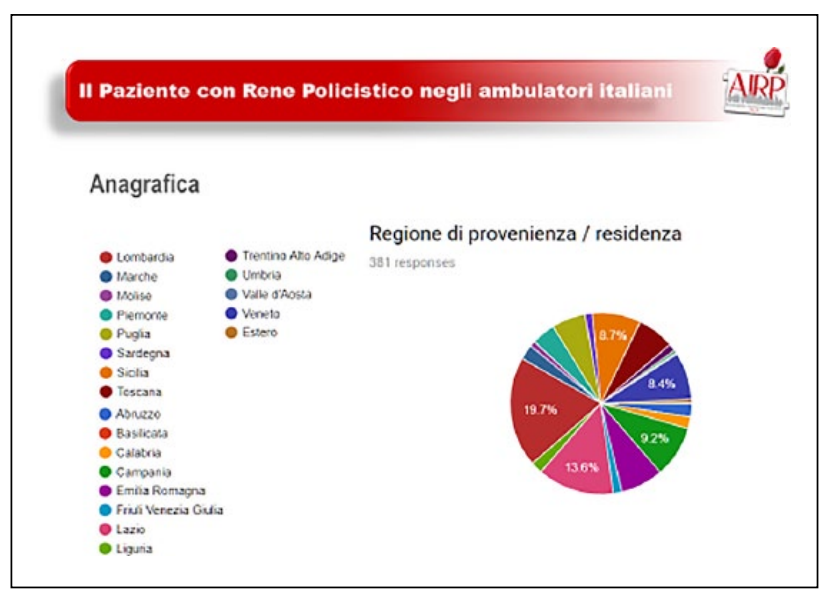

Un terzo dei pazienti che ha risposto al questionario è già stato sottoposto a trapianto (35.1 vs 64.9). Questo è un dato importante, perché sono soggetti che hanno l'esperienza sia della fase precedente che della fase successiva a tale procedura terapeutica e quindi con una visione completa di questo iter terapeutico.

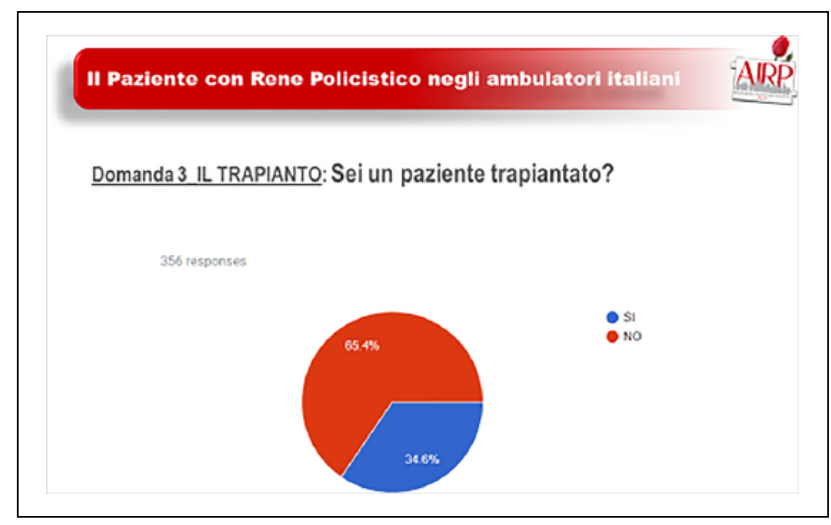

La maggior parte dei soggetti in lista vi è stata inserita nel momento in cui l'insufficienza renale è evoluta sino allo stadio V (filtrato glomerulare inferiore a 15), che è lo stadio in cui si rende necessaria una terapia sostitutiva, sia essa la dialisi od il trapianto stesso. In ambito nefrologico è dibattuto se sia meglio mettere in lista di trapianto i pazienti prima che giungano alla dialisi (trapianto pre-emptive). Pur a fronte di aspetti clinici positivi del trapianto pre-emptive, l'algoritmo recentemente elaborato dal Centro Nazionale Trapianti (Italian National Kidney Algorithm - INKA) tiene conto, nell'assegnare i reni, della durata della dialisi considerando prioritari, per aspetti etici, i pazienti che già da tempo sono in terapia sostitutiva.

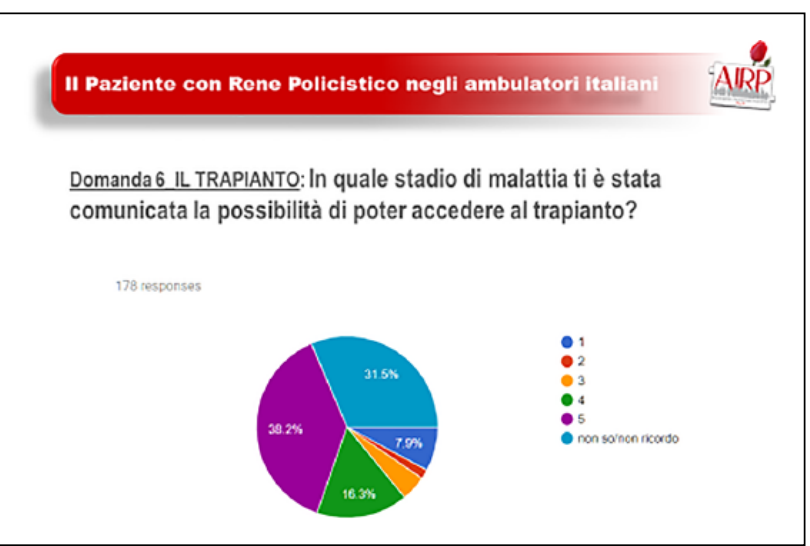

Il 75.4\% dei pazienti che ha risposto al questionario non è ancora inserito in una lista d'attesa di trapianto. Questo verosimilmente è determinato dal fatto che molti di loro non hanno ancora raggiunto lo stadio $\mathrm{V}$ dell'insufficienza renale, ma in altri casi è dovuto alla presenza di motivi che controindicano l'intervento e le terapie successive $(\mathrm{ad}$ esempio, severa malattia cardiovascolare grave, infezioni o tumori). 
Per alcuni dei pazienti il tempo d'attesa tra inserimento in lista e trapianto è stato inferiore all'anno, ma purtroppo per altri è stato più lungo.

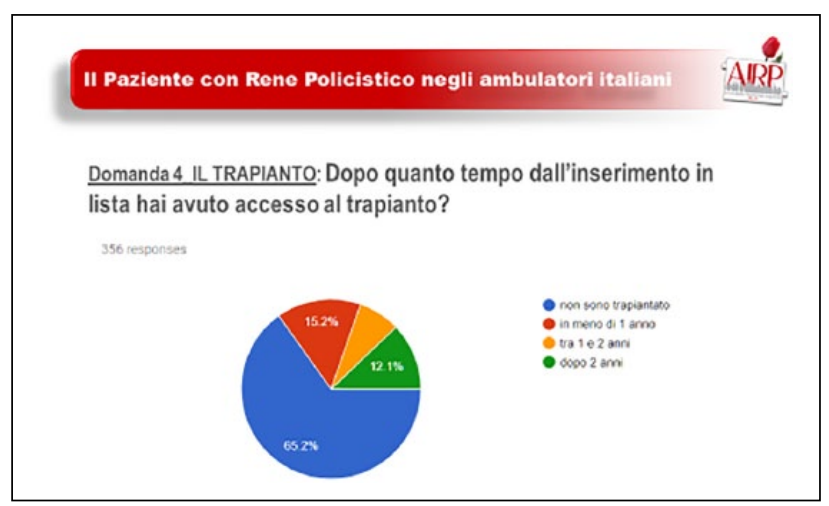

Le informazioni relative al trapianto vengono trasmesse in modo esaustivo al $94.9 \%$ dei pazienti indicando il ruolo fondamentale dei medici e del personale infermieristico in tale supporto al paziente ed alla sua famiglia.
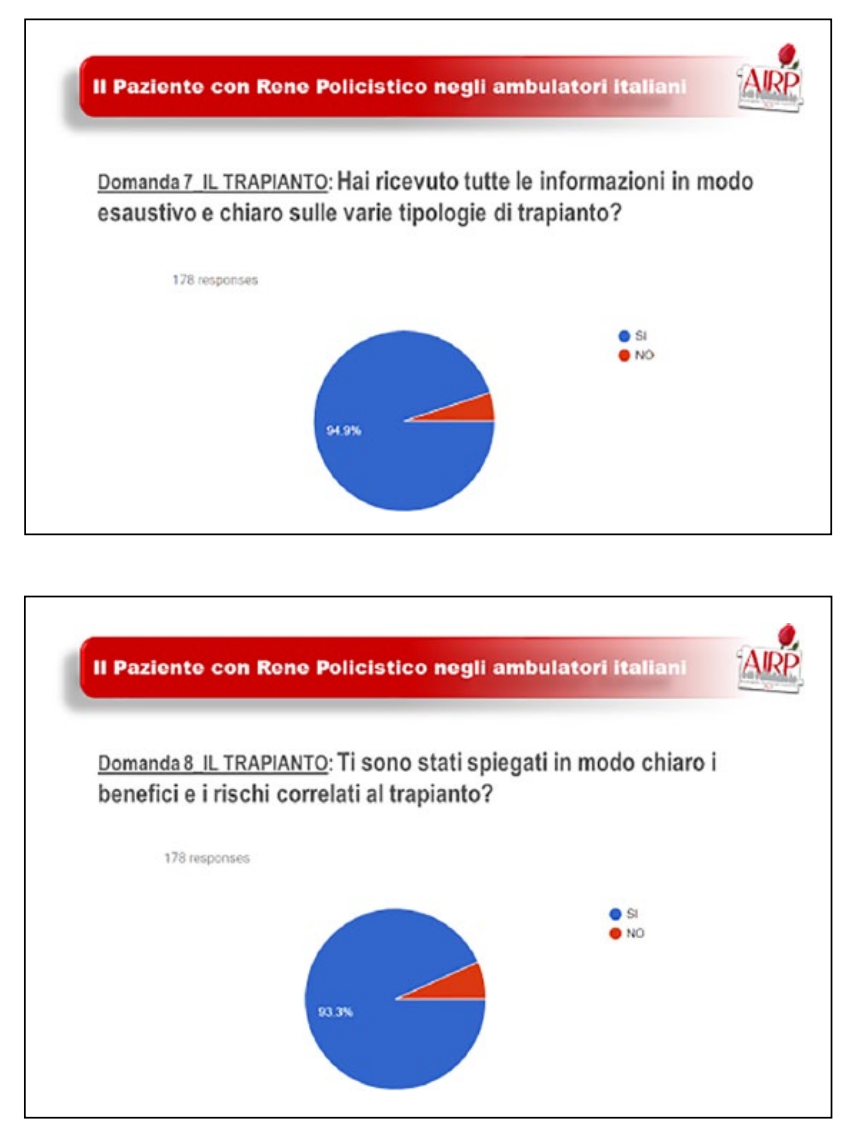

Una lacuna che meriterebbe di essere colmata è quella relativa all'aiuto da parte di uno psicologo, aiuto che la maggior parte dei pazienti non riceve.

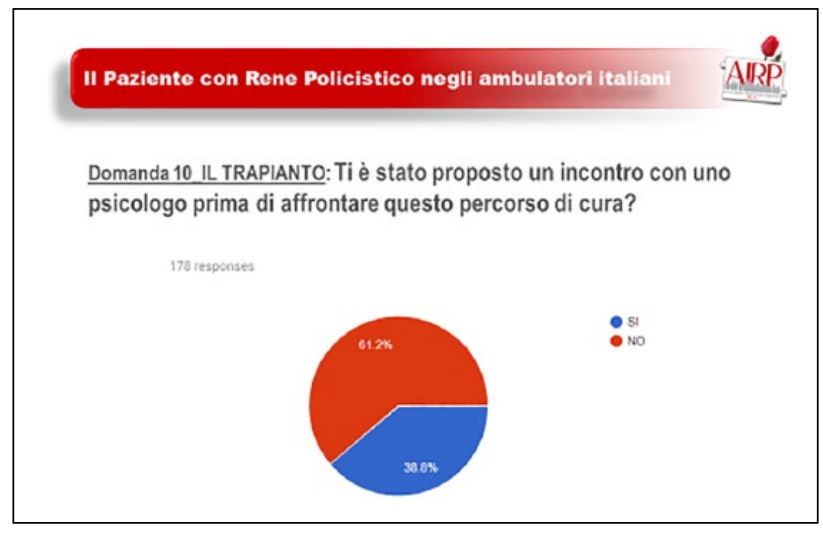

Uno degli aspetti di idoneità chirurgica al trapianto è l'indicazione o meno alla nefrectomia dei reni nativi. La necessità di procedere alla nefrectomia viene da vari fattori, tra cui la presenza di cisti complicate (infette e/o emorragiche) oppure le dimensioni dei reni talvolta tali da impedire, per questioni di ingombro, il trapianto stesso. Dalle risposte raccolte si evince che tale procedura non è stata necessaria nel 59.3\% dei soggetti prima del trapianto. In alcuni casi la nefrectomia può rendersi necessaria dopo il trapianto, in genere per il sopraggiungere di complicanze relative alla presenza delle cisti (ad esempio, infezioni delle cisti).

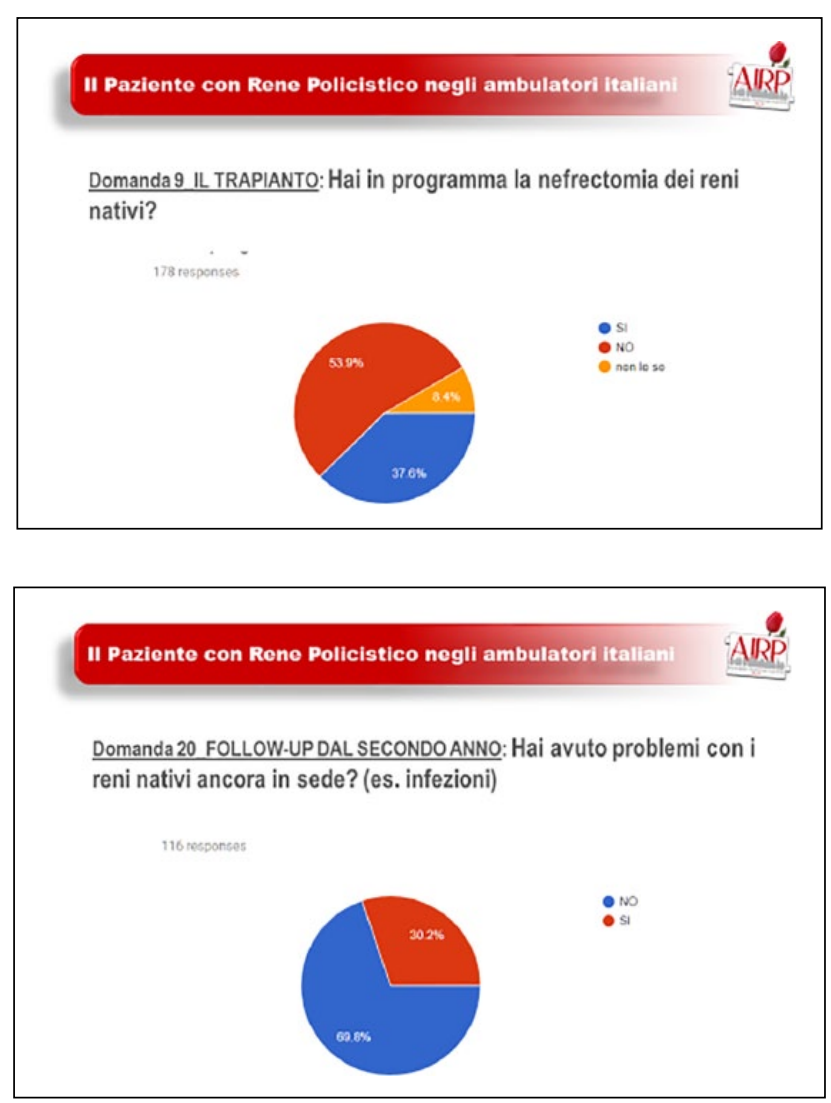




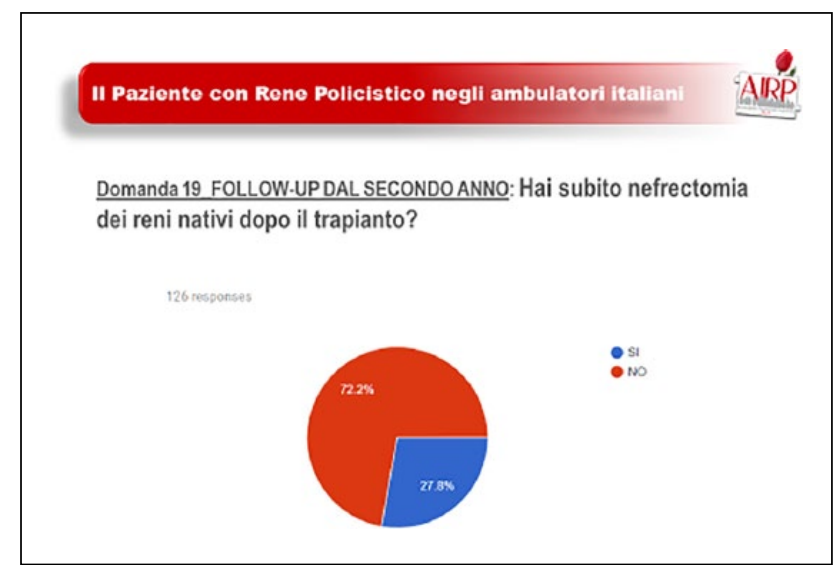

Un altro aspetto che viene valutato nel paziente con rene policistico è la presenza di aneurismi cerebrali. Il controllo della loro presenza ed il loro eventuale trattamento vengono effettuati generalmente prima del trapianto, anche se un attento monitoraggio e cura possono rendersi necessari anche dopo.

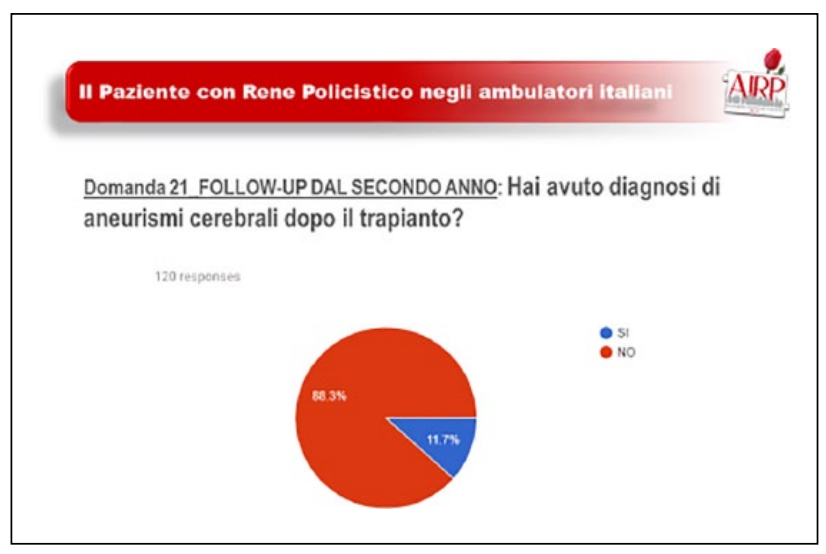

Una volta trapiantati, i pazienti vengono seguiti dai centri nefrologici di provenienza pur proseguendo, soprattutto nel primo anno, controlli anche presso i centri di trapianto a scadenze variabili in base al periodo postintervento od alle necessità cliniche. Tali controlli posttrapianto tendono a ridursi nel tempo pur mantenendosi presenti.

\section{Paziente con Rene Policistico negll ambulatori italia}

Domanda 23 FOLLOW-UPDALSECONDO ANNO: Da chi vieni seguito dopo il trapianto?

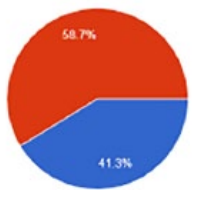

Domanda 11 PRIMO ANNO DAL TRAPIANTO: In media, ogni quanto ti rechi dal nefrologo/centro trapianti per le visite di controllo?

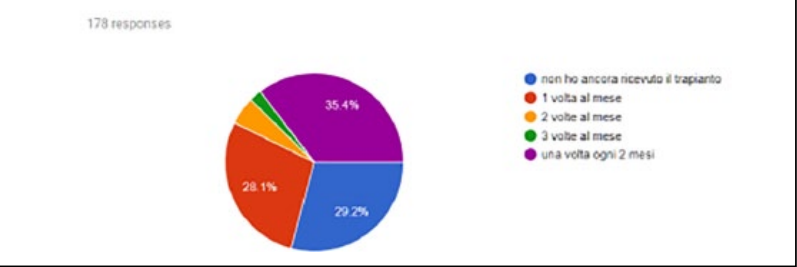

\section{Paziente con Rene Policistico negli ambulatori Itallani AIRP}

Domanda 16 FOLLOW.UPDAL SECONDOANNO: In media ogni quanto ti rechi dal nefrologo/centro trapianti per le visite di controllo?

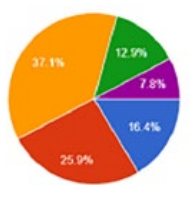

: ins wata almese

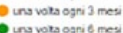
- ins vota arano

Ricevere un trapianto significa dover assumere la terapia immunosoppressiva. Fortunatamente la maggior parte dei pazienti ha riferito di tollerare abbastanza bene tale complesso trattamento.

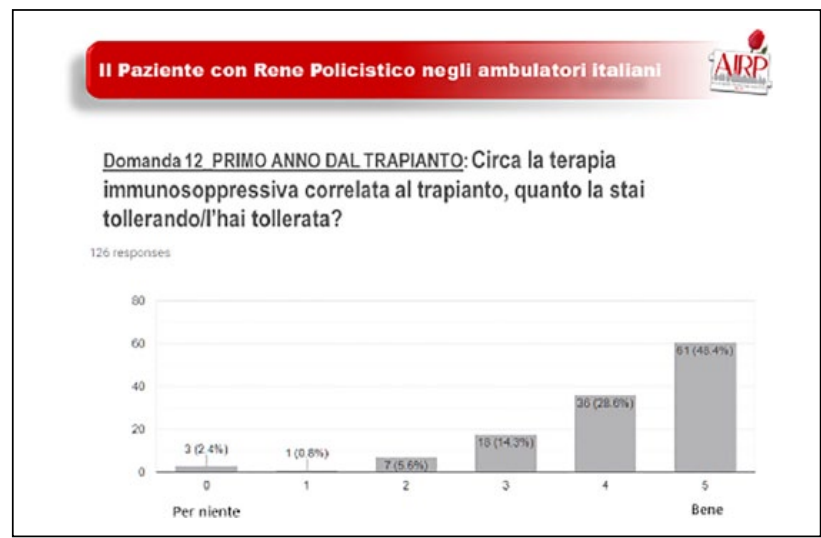

II Paziente con Rene Policistico negli ambulatori Italliar

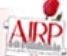

Domanda 17 FOLLOW.UPDAL SECONDO ANNO: Circa la terapia immunosoppressiva correlata al trapianto, quanto la stai tollerando/l'hai tollerata?

int responses

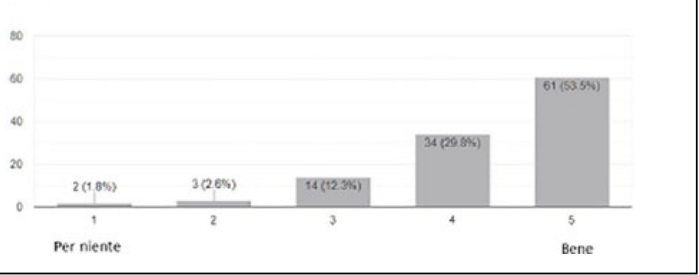


La maggior parte delle cause di modifica della posologia dei farmaci è stata determinata dalla necessità di adeguare il loro livello nel sangue o per ridurne gli effetti tossici, o per garantirne un'adeguata azione anti-rigetto. In alcuni casi, la modifica della terapia si è resa necessaria per la comparsa di effetti collaterali. La buona tollerabilità della terapia immunosoppressiva si mantiene anche dal secondo anno in poi con una riduzione dei casi in cui si rendono necessarie modifiche per la comparsa di effetti collaterali.
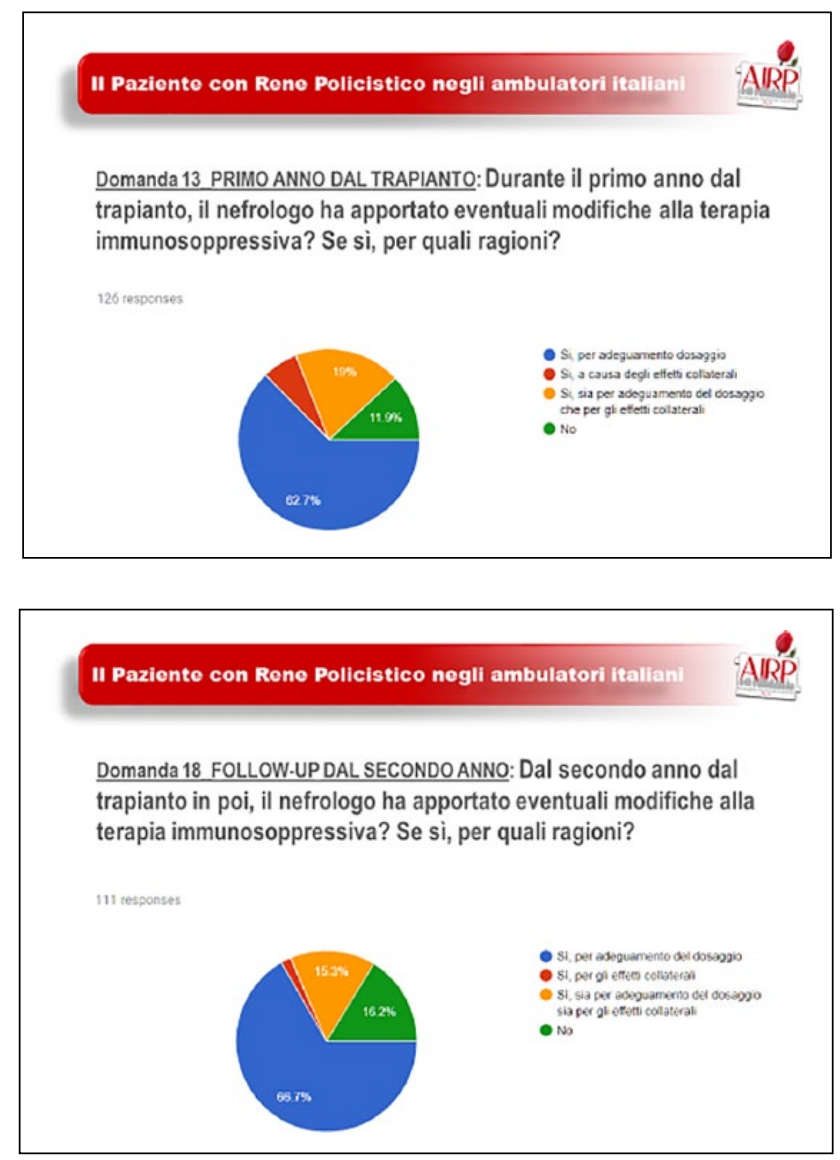

In alcuni casi, purtroppo, l'aderenza alle prescrizioni terapeutiche non è ottimale, perché la terapia non viene assunta con regolarità. Tale comportamento, purtroppo, espone al rischio di sviluppare anticorpi contro l'organo trapiantato inducendo un rigetto. ${ }^{4}$

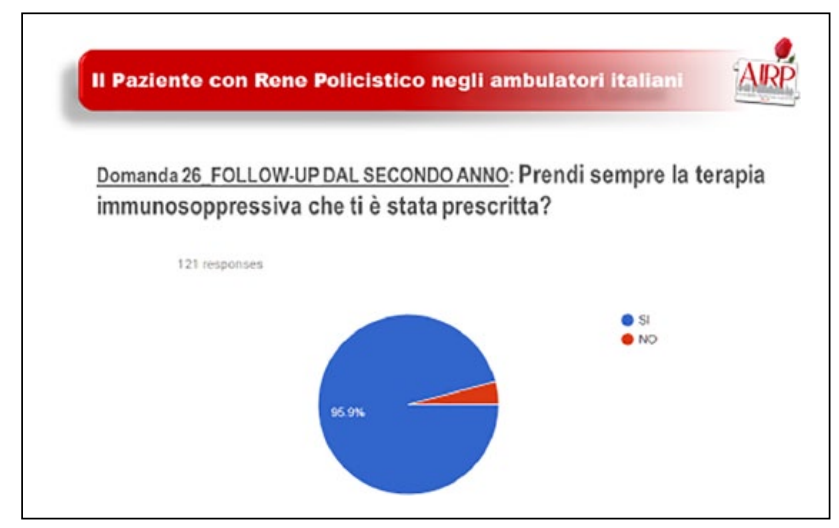

II Pazionto con Rone Policistico negli ambulatori italiat

Domanda 27 FOLLOW.UPDALSECONDO ANNO: Ti è mai capitato di dimenticarla?

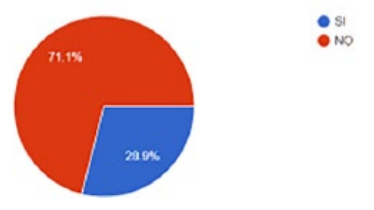

Uno degli effetti collaterali della terapia immunosoppressiva è la riduzione delle difese immunitarie nei confronti delle infezioni. Seppur il $43.3 \%$ dei pazienti riferisce di non aver avuto infezioni, altri hanno necessitato di cure per infezioni batteriche, virali o per entrambe anche a distanza dal trapianto.

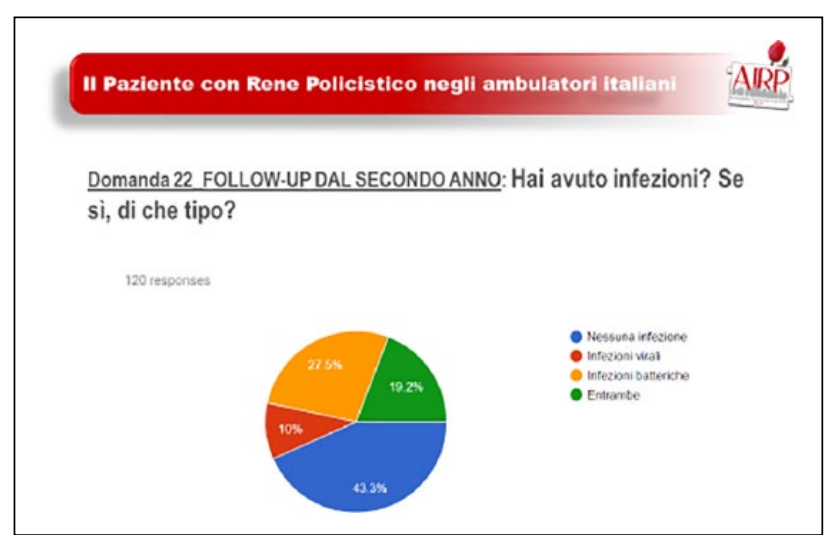

Per le possibili complicanze della terapia immunosoppressiva e per garantire il più a lungo possibile una buona funzione del rene trapiantato, tutti i pazienti dovrebbero essere sottoposti ad esami di controllo periodici. Questo è quanto in effetti sembra avvenire.

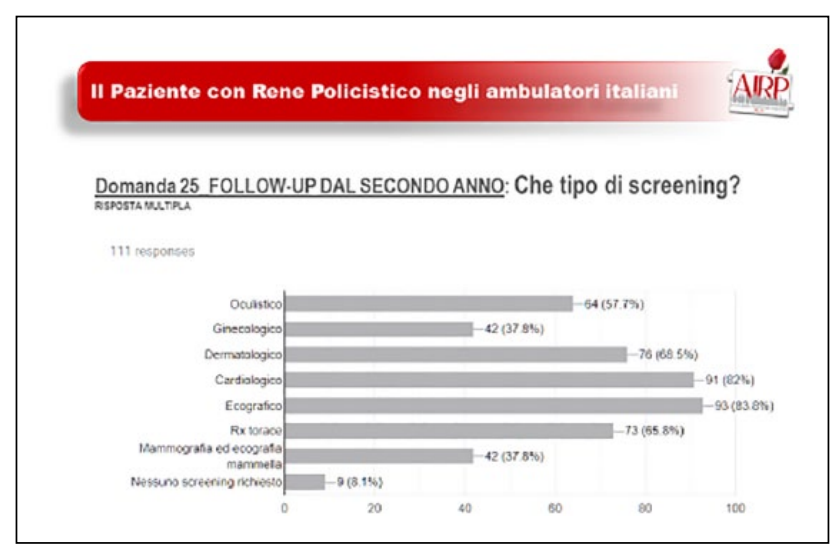

Nel primo anno dopo il trapianto i pazienti considerano migliorata la loro qualità di vita per quanto concerne soprattutto la vita sociale e familiare. Per la metà dei 
soggetti migliora anche la vita di coppia e quanto concerne l'attività lavorativa. Per 1'84\% dei soggetti che hanno risposto alle domande relative alla qualità di vita non vi sono in genere grosse limitazioni.
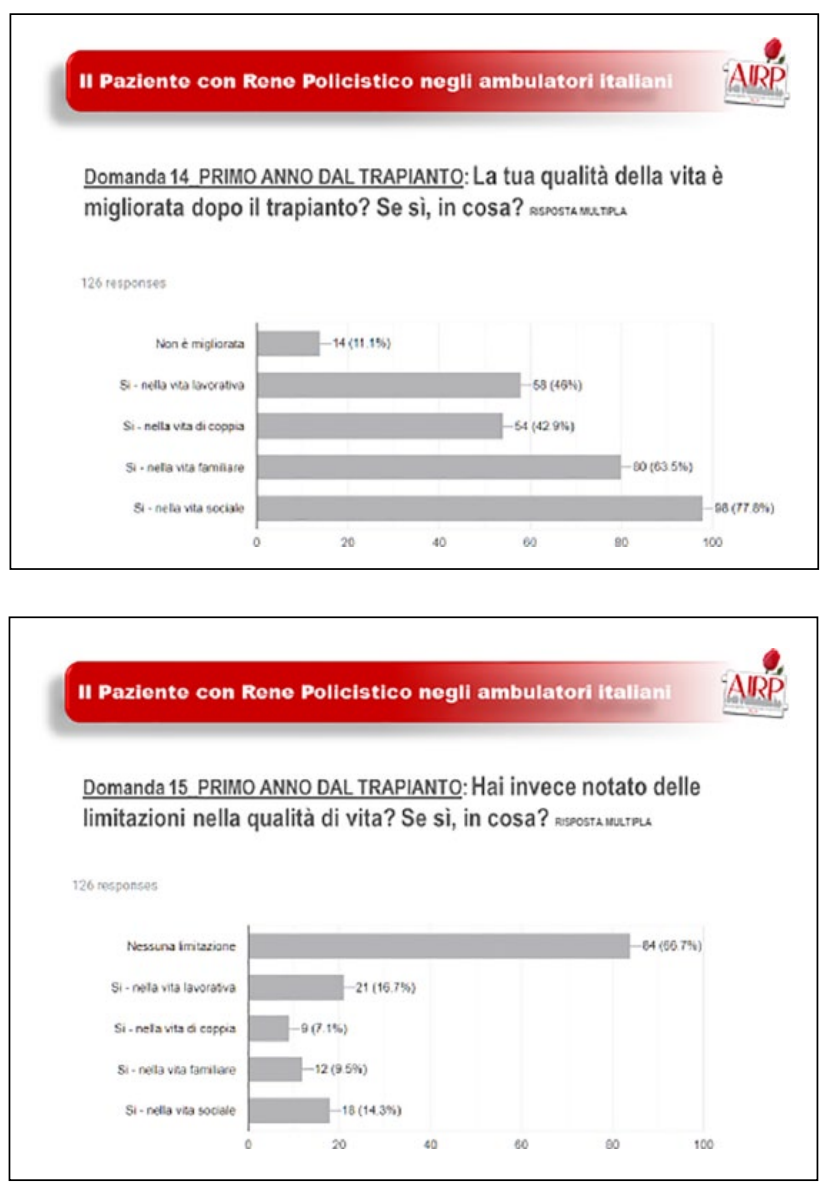

Solo la metà dei soggetti, però, svolge attività fisica costantemente.

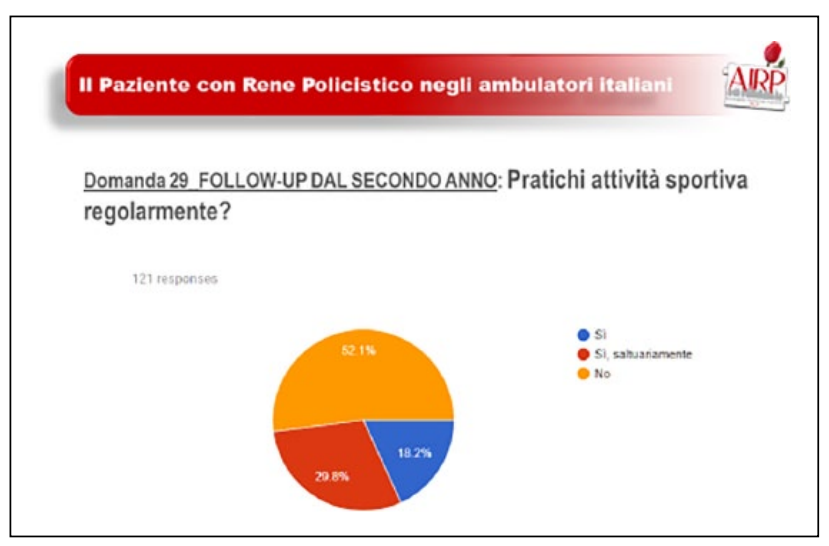

Il trapianto non preclude la possibilità di aver figli, anche se questo deve avvenire sotto stretto monitoraggio ostetrico-ginecologico e nefrologico. Il fatto che il paziente affetto arrivi al trapianto in età relativamente avanzata condiziona, verosimilmente, la bassa percentuale di soggetti che hanno indicato di aver avuto figli dopo il trapianto.

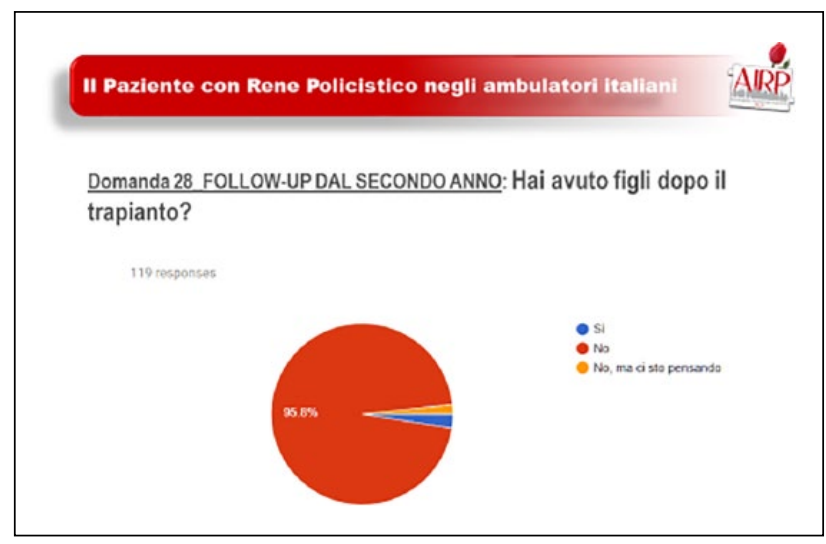

\section{Testimonianze}

Bisognerebbe creare una precedenza per chi si sottopone alle visite per la messa in lista. Un anno e mezzo per completare gli esami è una cosa disumana, un calvario!

Il problema più grosso dal punto di vista della gestione del paziente l'ho trovato nell'immediato post-trapianto perché non sentivo di essere seguita con attenzione visto che il medico di base e i medici del centro trapianti locale rimandavano le mie cure all'ospedale dove era stato eseguito il trapianto, dove non sempre riuscivo ad avere risposte in tempi celeri e spesso c'era un rimbalzo di responsabilità.

Sono stata inserita nella lista trapianti del Lazio prima della dialisi. Fortunatamente mio marito è risultato idoneo a donarmi un rene, per cui ho potuto evitare di andare in dialisi. Dopo 2 mesi dal trapianto ho fatto la nefrectomia dei reni nativi.

Trapianto pre-empty. Nessun disturbo dopo il trapianto. Prima solo ematuria senza dolori particolari.

Fortunato. . .

Ho avuto 2 trapianti, il primo dopo 9 mesi di dialisi, il secondo dopo 12 anni. Sono passati 9 anni dall'ultimo trapianto, attualmente sto bene, $i$ dati delle analisi sono positivi, consiglio a tutti grande attenzione nel primo anno dopo il trapianto e rigorosa attenzione alla terapia.

La mia esperienza sino a qui è sicuramente positiva. L'assunzione regolare dei farmaci comporta diversi effetti collaterali che hanno come conseguenza diretta quella di complicare la vita di tutti i giorni e il modo di relazionarsi con il prossimo. Mi riferisco in particolare al cortisone che oltre a causare effetti collaterali diretti, come gastrite etc, provoca sbalzi di umore che creano difficoltà come già citato nella gestione delle relazioni umane. In ogni caso il trapianto dà la possibilità a persone che soffrono e che si sentono ormai ai margini di riappropriarsi della propria vita ridando loro dignità e nuovi stimoli. 


\section{Considerazioni finali}

Molti dei pazienti che hanno risposto al questionario hanno voluto raccontare, in poche righe, la loro esperienza. È da queste testimonianze che partiamo per alcune considerazioni finali che considereranno aspetti positivi e negativi della nostra realtà italiana.

L'iter clinico-strumentale per definire l'idoneità al trapianto è complesso, perché prevede l'esecuzione di numerosi accertamenti. Purtroppo, ci sono pazienti che impiegano molti mesi a completare gli esami e questo va a ritardare il loro ingresso in lista di trapianto. Spesso i Centri Trapianto richiedevano, inoltre, accertamenti in parte diversi. Se è vero che al paziente è riconosciuta un'esenzione dal pagamento del ticket (codice 050), non esiste però ancora un percorso standardizzato e/o prioritario valido per tutte le Nefrologie o Centri Trapianto.

$\mathrm{Ci}$ sono però in atto tentativi per migliorare tale situazione, quale ad esempio quello fatto dalla Società Italiana di Nefrologia con la Società Italiana Trapianto d'organo. Le due società, sotto l'egida del Centro Nazionale trapianti, hanno infatti lavorato congiuntamente per identificare gli esami di primo livello necessari per l'immissione in lista, ed hanno redatto una cartella di iscrizione che verrà utilizzata, uniformemente, in Italia.

Una problematica che è emersa è quella relativa ai rapporti tra pazienti e medici e tra specialisti diversi. Per giungere al trapianto e per goderne a lungo i risultati è fondamentale la relazione tra i pazienti ed il personale delle Nefrologie e dei Centri Trapianto. Il paziente deve essere adeguatamene accompagnato attraverso le varie fasi che si succedono nel tempo e trovare negli specialisti gli alleati pronti a garantirgli le indicazioni terapeutiche più idonee e ad intervenire di fronte alle possibili necessità.

Il follow-up post-trapianto deve prevedere una collaborazione stretta tra Nefrologo di riferimento del paziente e medico del Centro Trapianti perché entrambi, per loro competenze e peculiarità, devono garantire l'attento monitoraggio delle condizioni del paziente.

Grazie ai progressi scientifici e tecnologici, oggi possiamo utilizzare organi che un tempo non avremmo potuto trapiantare. Oggi possiamo trapiantare, con successo, organi da donatori anziani o da deceduti per cause cardiache. Possiamo analizzare e trattare i reni prima di trapiantarli, grazie alle analisi istologiche ed all'uso delle macchine di perfusione, garantendone meglio la qualità.

Purtroppo, la realtà italiana è però caratterizzata da un numero elevato di pazienti in attesa di trapianto rispetto alla disponibilità di organi. Come già commentato, raramente è possibile giungere al trapianto pre-emptive che è uno dei desideri maggiori dei pazienti.

Ma una possibilità è il trapianto da donatore vivente.

Naturalmente anche per il paziente con ADPKD questa è una valida opzione in presenza di un donatore vivente non consanguineo. In realtà, previa attenta valutazione clinica, è talvolta possibile anche la donazione da donatore consanguineo. ${ }^{5}$

Dalle testimonianze dei pazienti emergono le problematiche relative agli effetti collaterali dei farmaci ed alla necessità di un rigore nell'aderire alle indicazioni terapeutiche, situazioni che naturalmente gravano sugli aspetti della vita quotidiana.

Per concludere, vogliamo però utilizzare proprio una di queste testimonianze, perché è riuscita a sintetizzare al meglio quello che il trapianto di rene può significare:

"Grazie al trapianto la mia vita sociale e familiare è cambiata in modo positivo. Ho avuto, e forse ne avrò ancora, problemi dovuti ai farmaci, ma grazie alla mia forza di volontà e alla mia famiglia sono riuscito a superarli. Grazie al mio donatore."

\section{Dichiarazione di assenza di conflitto di interessi}

Gli Autori dichiarano di non avere conflitti di interessi.

\section{Finanziamenti}

Gli Autori dichiarano di non aver ricevuto finanziamenti specifici da qualsiasi ente nei settori pubblico, privato o senza fini di lucro.

\section{Bibliografia}

1. Mosconi G, Persici E, Cuna V, et al. Renal transplant in patients with polycystic disease: the Italian experience. Transplantation Proceedings 2013; 45:2635-2643.

2. ERA-EDTA Registry: Transplantation. Sept 2016, vol 100, number 9 .

3. Franz KA and Reubi FC. Rate of functional deterioration in polycystic kidney disease. Kidney international 1983; 23(3):526-529.

4. Sellares J, de Freitas DG, Mengel M, et al. Understanding the causes of kidney transplant failure: the dominant role of antibody-mediated rejection and nonadherence. American Journal of Transplantation 2012; 12:388-399.

5. Kanaan N, Devuyst O, Pirson Y, et al. Renal transplantation in autosomal dominant polycystic kidney disease. Nat Rev Nephrol 2014; 10:455-465. 